\title{
Chromosomal instability as a prognostic marker in cervical cancer
}

\author{
Christine How ${ }^{1}$, Jeff Bruce ${ }^{1}$, Jonathan So ${ }^{2,3}$, Melania Pintilie ${ }^{4}$, Benjamin Haibe-Kains ${ }^{1,7}$, Angela Hui ${ }^{1}$, Blaise A Clarke ${ }^{5}$, \\ David W Hedley ${ }^{1,6}$, Richard P Hill ${ }^{1}$, Michael Milosevic ${ }^{2,3}$, Anthony Fyles ${ }^{2,3}$ and Fei-Fei Liu $u^{1,2,3^{*}}$
}

\begin{abstract}
Background: Cervical cancer is the third most common cancer in women globally, and despite treatment, distant metastasis and nodal recurrence will still develop in approximately $30 \%$ of patients. The ability to predict which patients are likely to experience distant relapse would allow clinicians to better tailor treatment. Previous studies have investigated the role of chromosomal instability (CIN) in cancer, which can promote tumour initiation and growth; a hallmark of human malignancies. In this study, we sought to examine the published CIN70 gene signature in a cohort of cervical cancer patients treated at the Princess Margaret (PM) Cancer Centre and an independent cohort of The Cancer Genome Atlas (TCGA) cervical cancer patients, to determine if this CIN signature associated with patient outcome.
\end{abstract}

Methods: Cervical cancer samples were collected from 79 patients, treated between 2000-2007 at the PM, prior to undergoing curative chemo-radiation. Total RNA was extracted from each patient sample and analyzed using the GeneChip Human Genome U133 Plus 2.0 array (Affymetrix).

Results: High CIN70 scores were significantly related to increased chromosomal alterations in TCGA cervical cancer patients, including a higher percentage of genome altered and a higher number of copy number alterations. In addition, this same CIN70 signature was shown to be predictive of para-aortic nodal relapse in the PM Cancer Centre cohort.

Conclusions: These findings demonstrate that chromosomal instability plays an important role in cervical cancer, and is significantly associated with patient outcome. For the first time, this CIN70 gene signature provided prognostic value for patients with cervical cancer.

Keywords: Cervical cancer, Chromosomal instability, CIN, mRNA, TCGA

\section{Background}

Chromosomal instability (CIN), a condition in which cells change their chromosomal content at a high rate, is a consistent feature of the majority of solid tumours $[1,2]$. It has long been postulated that chromosomal imbalance plays a role in tumourigenesis, since aneuploid karyotypes were first observed in cancer cells over a century ago [3]. Since then, evidence has shown that CIN promotes tumour initiation and growth [4-7]. In patient tumours, it has been demonstrated that CIN increases with increasing tumour grade as well as invasiveness

\footnotetext{
* Correspondence: Fei-Fei.Liu@rmp.uhn.on.ca

'Princess Margaret Cancer Centre, University Health Network, Toronto, ON, Canada ${ }^{2}$ Department of Radiation Oncology, Princess Margaret Cancer Centre, University Health Network, Toronto, ON, Canada

Full list of author information is available at the end of the article
}

[8-11]. Some studies have alluded to the clinical value of CIN in human cancers $[8,12]$, although therapeutic targeting of CIN remains in its infancy [13].

Using a computational approach to identify specific genes whose expression was consistently correlated with total functional aneuploidy across multiple cancer types, Carter et al. developed a gene expression signature of CIN, the CIN70, which could predict patient survival and prognosis [14]. Over-expression of this CIN70 signature was predictive of poor clinical outcome in 12 datasets representing six types of tumour: lymphoma, lung adenocarcinoma, glioma, medulloblastoma, mesothelioma, and breast cancer [15-26]. In this study, we sought to examine CIN in cervical cancer and determine 
if the CIN70 signature could also be used to predict clinical outcome in patients with cervical cancer.

Globally, cervical cancer is the third most common cancer in women [27]. Although there has been a decrease in cervical cancer incidence and mortality over the past thirty years in the United States, the five-year survival rate remains below $40 \%$ for stage III and stage IV patients [28]. Furthermore, distant metastasis and lymph node recurrence occurs in approximately $30 \%$ of patients following primary treatment [29]. The ability to predict which patients are likely to experience distant relapse would allow clinicians to better tailor patient therapy.

In this current study, the CIN70 signature was investigated in a cohort of cervical cancer patients treated at the Princess Margaret (PM) Cancer Centre $(n=79)$, and an independent cohort of TCGA cervical cancer patients $(n=130)$. CIN70 score was found to be significantly associated with chromosomal alterations and paraaortic distant relapse in patients. Altogether, these findings provide insight into the role of CIN in cervical cancer and show that CIN can harbour clinical value for patients.

Table 1 Clinical parameters of the Princess Margaret Cancer Centre cohort

\begin{tabular}{|c|c|}
\hline & $n=79$ \\
\hline \multicolumn{2}{|l|}{ Age (years) } \\
\hline Median & 48 \\
\hline Range & $26-84$ \\
\hline \multicolumn{2}{|l|}{ Tumour size } \\
\hline$\leq 5 \mathrm{~cm}$ & $48(61 \%)$ \\
\hline$>5 \mathrm{~cm}$ & $31(39 \%)$ \\
\hline \multicolumn{2}{|l|}{ FIGO stage } \\
\hline $\mathrm{IA}$ & 0 \\
\hline $\mathrm{IB}$ & $24(30 \%)$ \\
\hline$\| \mathrm{A}$ & $2(3 \%)$ \\
\hline$\| \mathrm{B}$ & $35(44 \%)$ \\
\hline IIIA & 0 \\
\hline$\| \mathrm{I} B$ & $18(23 \%)$ \\
\hline \multicolumn{2}{|c|}{ Pelvic or para-aortic node involvement } \\
\hline Positive & $25(32 \%)$ \\
\hline Equivocal & $15(19 \%)$ \\
\hline Negative & $39(49 \%)$ \\
\hline \multicolumn{2}{|l|}{ Overall survival } \\
\hline Deaths & $24(31 \%)$ \\
\hline \multicolumn{2}{|l|}{ Disease-free survival } \\
\hline Relapses or deaths & $28(35 \%)$ \\
\hline \multicolumn{2}{|l|}{ Follow-up (years) } \\
\hline Median & 6.0 \\
\hline Range & $0.7-10.6$ \\
\hline
\end{tabular}

\section{Methods}

Ethics statement

Written informed consent was obtained from all human subjects, according to a protocol (09-0245-T) approved for this study by the University Health Network Research Ethics Board.

\section{Clinical specimens}

Frozen pre-treatment cancer samples were collected from 79 patients with cervical cancer, prior to undergoing curative chemo-radiation, consisting of external-beam radiotherapy to the primary cervical tumour and pelvic lymph nodes ( 45 to $50 \mathrm{~Gy}$ total, in 1.8 to 2 Gy daily fractions using 18 or 25 Megavolt photons), combined with weekly cisplatin ( $40 \mathrm{mg} / \mathrm{m}^{2}$ total, 5 doses). These patients were treated at the PM Cancer Centre between 2000 and 2007. Patients were staged using the FIGO (International Federation of Gynecologists and Obstetricians) system, with additional clinical information gathered using computed tomography (CT) scans of the abdomen and pelvis, as well as magnetic resonance imaging (MRI) of the pelvis to assess local and lymphatic disease. Pelvic and para-aortic lymph nodes were classified as positive for metastatic disease if the MRI short-axis dimension was $>1 \mathrm{~cm}$, and equivocal if it was between 8 to $10 \mathrm{~mm}$.

The frozen biopsy specimens were placed in a storage medium (optimal cutting temperature (OCT) compound) for histopathologic examination, then flash-frozen in liquid nitrogen. H\&E-stained tissue sections were cut from the OCT-embedded material, and evaluated by a

Table 2 Clinical parameters of TCGA cohort

\begin{tabular}{ll}
\hline & $\boldsymbol{n}=\mathbf{1 3 5}$ \\
\hline Age (years) & 46 \\
Median & $21-88$ \\
Range & \\
FIGO stage & $2(1.5 \%)$ \\
IA & $82(60.7 \%)$ \\
IB & $11(8.2 \%)$ \\
IIA & $11(8.2 \%)$ \\
IIB & 0 \\
IIIA & $19(14.1 \%)$ \\
IIIB & $1(0.7 \%)$ \\
IVA & $3(2.2 \%)$ \\
IVB & $6(4.4 \%)$ \\
N/A & \\
Overall survival & $19(14 \%)$ \\
Deaths & \\
Follow-up (years) & 0.36 \\
Median & $0-14.7$ \\
Range &
\end{tabular}


gynecology oncology pathologist (B. Clarke). The total cell content (stroma and tumour cells) was estimated for all tissue samples using a light microscope, and only samples containing at least $70 \%$ tumour cells were considered for further analysis. Flash-frozen normal cervix tissues obtained from 11 patients who underwent total hysterectomy for benign causes served as the normal comparators.

\section{Sample processing}

Two sections of 50- $\mu$ m thickness were cut from the OCTembedded flash-frozen tissues and placed in a nuclease-free

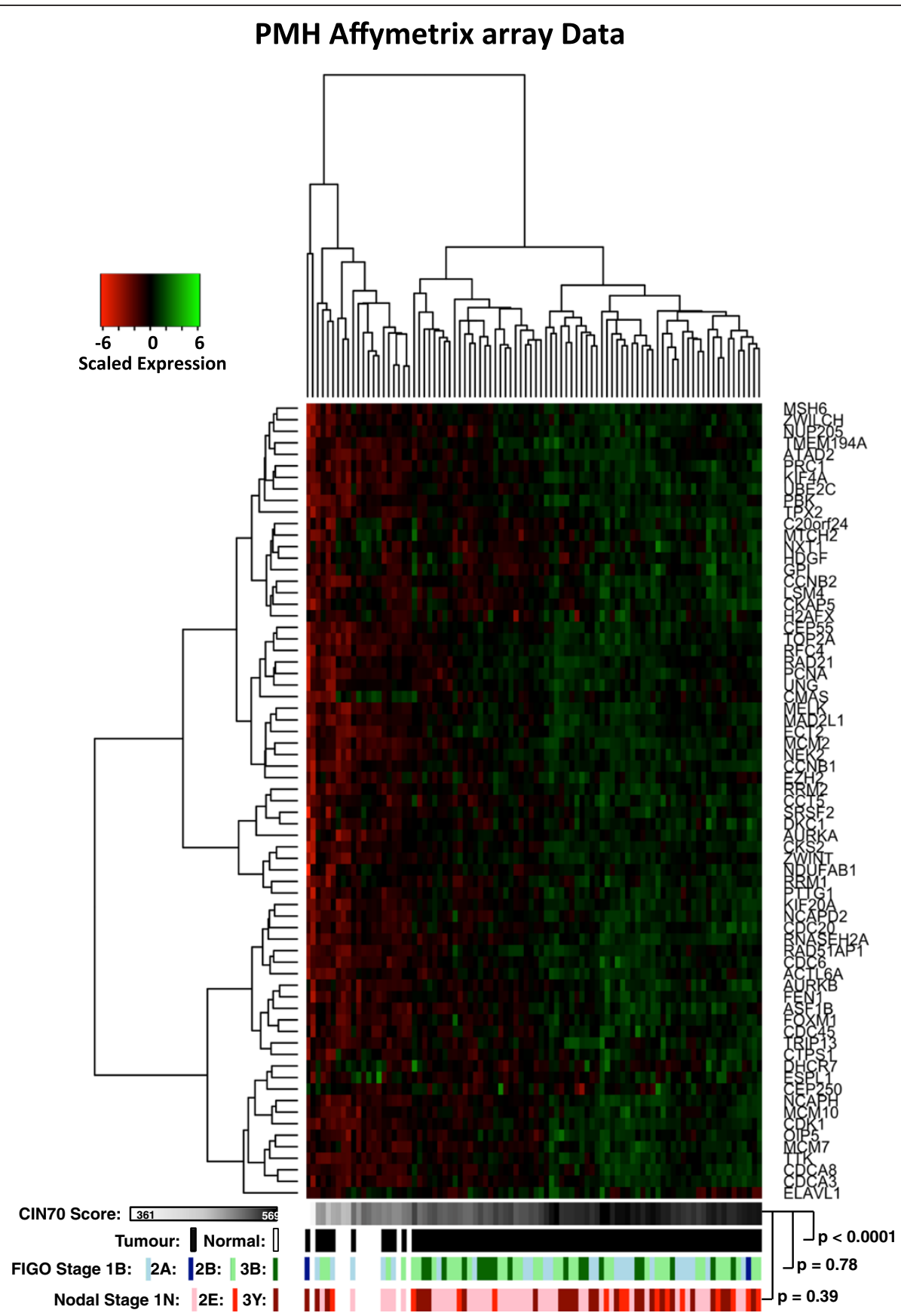

Figure 1 PM Cancer Centre Affymetrix heat map. Hierarchically clustered heat map showing scaled expression of CIN70 genes in cervix tumour $(n=79)$ and normal $(n=11)$ tissues, compared to CIN70 score (white to black scale). Comparisons are also made with FIGO stage (1B, 2A, 2B, and $3 \mathrm{~B})$, and nodal stage ( $1 \mathrm{~N}=$ negative, $2 \mathrm{E}=$ equivocal, $3 \mathrm{Y}=$ positive). P-values refer to relationship between CIN70 scores with tumour:normal, FIGO stage, and Nodal stage. 
microtube. Total RNA was isolated using the Norgen Total RNA Purification Kit (Norgen Biotek), according to the manufacturer's instructions. Gene expression was measured with the GeneChip Human Genome U133 Plus 2.0 array (Affymetrix).

\section{Data analysis}

Affymetrix array data were pre-processed using the Robust Multi-array Average robust-multi array algorithm [30] in the $\mathrm{R}$ statistical environment with the affy package [31]. CIN70 score was calculated by summing the normalized expression of each gene in the CIN70 signature. For genes with more than one mapped probe set on the array, the probe set with the highest variance across samples was selected.

Level 3 copy-number (SNP 6.0 arrays; Affymetrix), geneexpression (RNA-Seq; Illumina) and somatic mutation (Exome-Seq; Illumina) data were downloaded from the

\section{TCGA RNA-Seq Data}

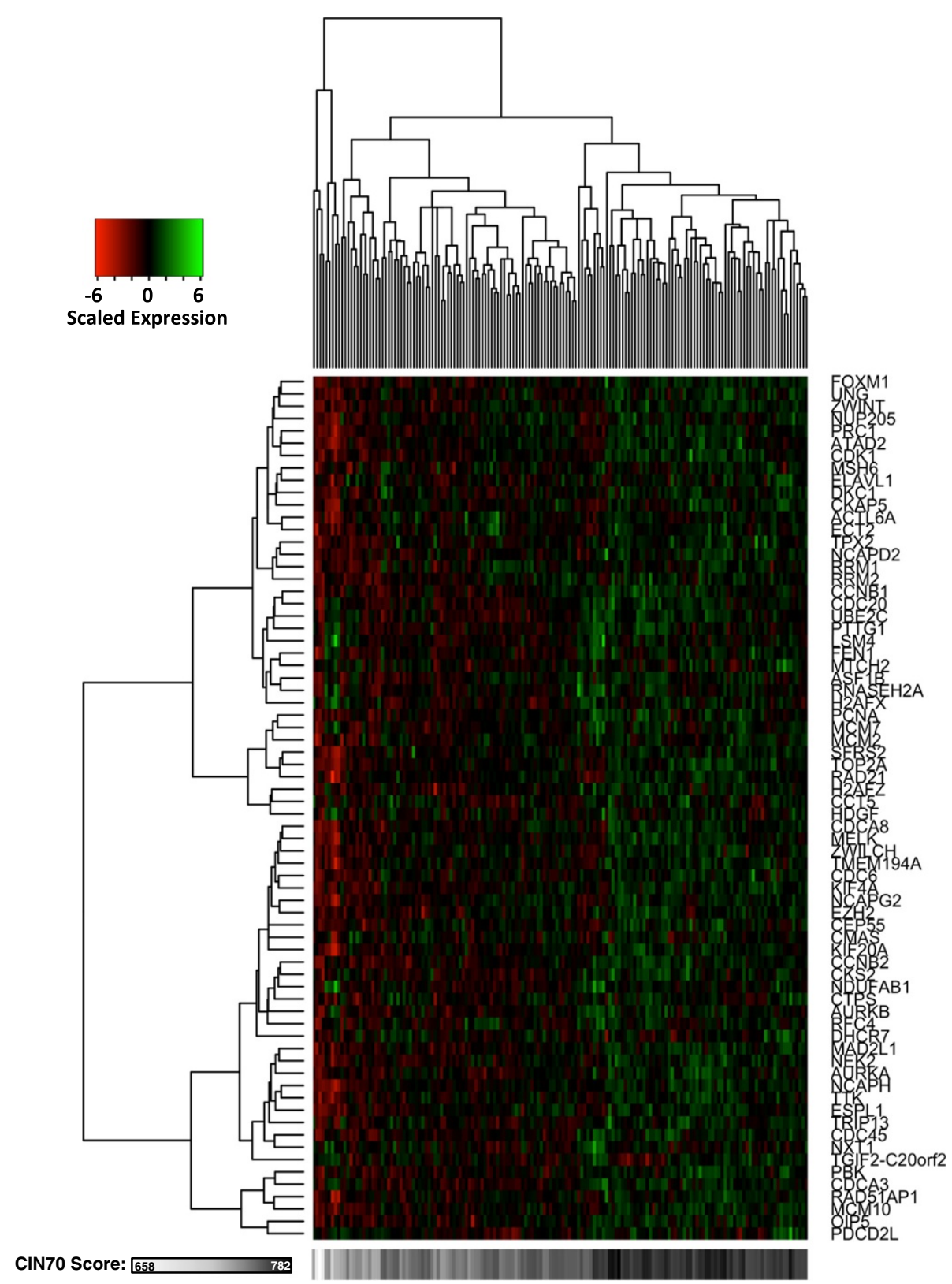

Figure 2 TCGA RNA-Seq heat map. Hierarchically clustered heat map showing scaled expression of CIN70 genes in TCGA cervix tumour tissues $(n=130)$, compared to CIN70 score. 
Broad GDAC Firehose (http://gdac.broadinstitute.org/), and analyzed in the $\mathrm{R}$ statistical environment. CIN70 score was calculated again by summing the normalized expression of each gene in the CIN70 signature. The number of copy-number alterations was calculated using segmented copy-number data, whereby segments with a mean $\log 2$ copy-number ratio value
$>0.2$ or $<-0.2$ were deemed altered [32,33]. Relatedly, percent genome altered was calculated by adding the length of each "altered" segment, divided by the total length of the genome analyzed. The number of mutations corresponded to somatic coding mutations were called using TCGA's Exome-Seq analysis pipeline.

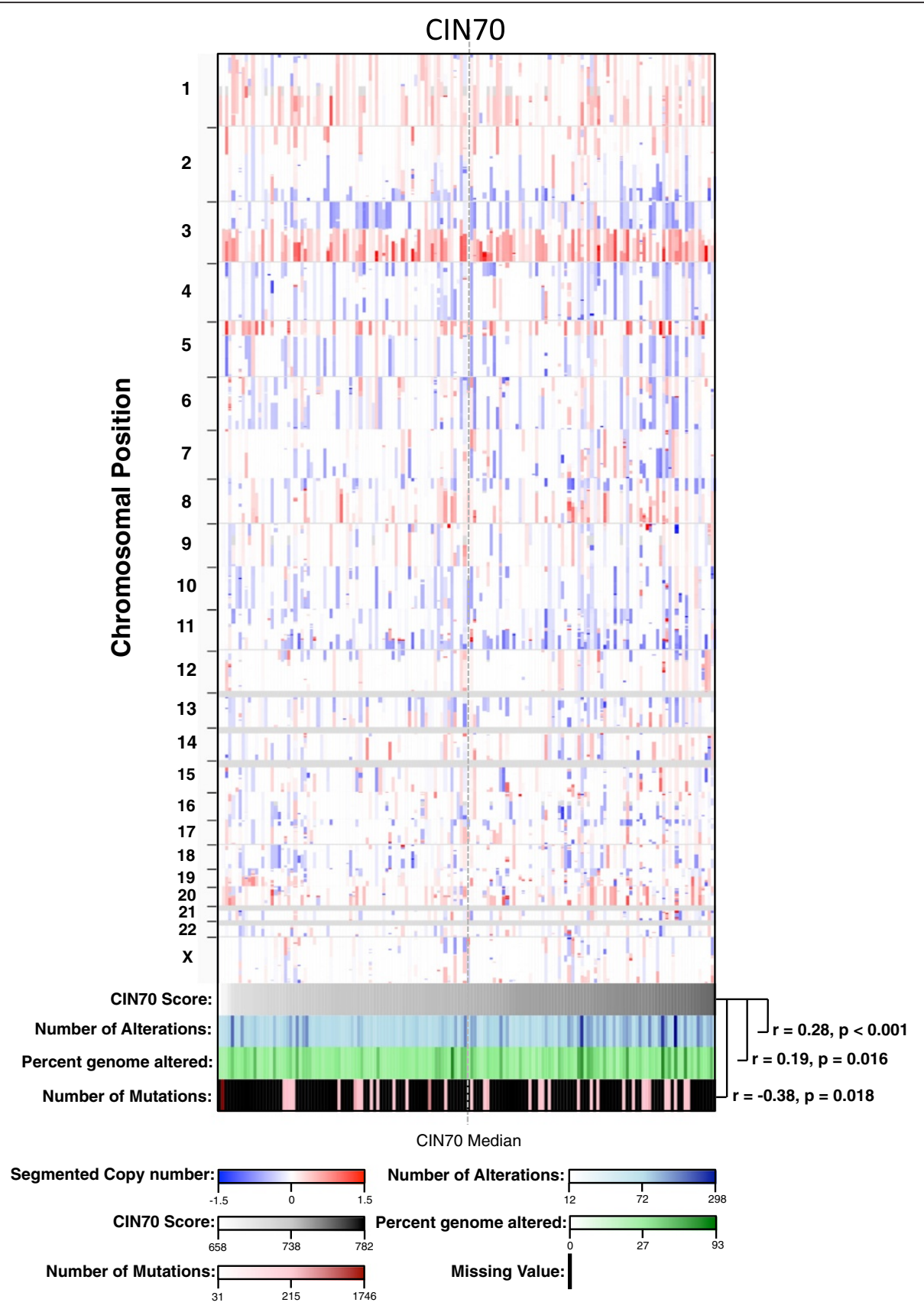

Figure 3 Chromosomal alterations in TCGA cervix cancer tissues. Copy number alterations (top) in TCGA cervical cancer patients, compared to CIN70 score (white to black scale), number of alterations (white to blue scale), percent genome altered (white to green scale), and number of mutations (white to red scale). Spearman's correlation coefficient ( $r$ ), and P-values are shown for each of the respective comparisons. 


\section{Survival analysis}

For each patient in the PM Cancer Centre cohort $(n=79)$, a risk score was calculated using the published CIN70 signature [14] and Affymetrix gene expression data. Risk scores were dichotomized at the median $($ CIN70 $=7.57)$ and the cohort was divided into low and high-risk groups. Curves for overall survival (OS), disease-free survival (DFS), probability of local relapse, and probability of distant relapse were plotted according to the KaplanMeier method, with p-values determined using the Wald test.

\section{Results}

PM and TCGA cohorts showed a distinct expression pattern of CIN70 genes according to CIN70 score

The clinical characteristics of the 79 PM Cancer Centre and 130 TCGA patients are shown in Tables 1 and 2, respectively. A heat map of scaled expression of the CIN70 genes showed a distinct expression pattern in patients according to CIN70 score (Figure 1). As expected, normal and tumour cervix samples had significantly different
CIN70 scores $(\mathrm{p}<0.0001)$. Interestingly, CIN70 score was not significantly associated with FIGO stage $(\mathrm{p}=0.78)$ or nodal stage $(\mathrm{p}=0.39)$. TCGA cervical cancer patients showed a similar expression pattern of the CIN70 genes according to CIN70 score (Figure 2).

\section{High CIN70 score was related to increased chromosomal alterations}

A heat map of TCGA patients (Figure 3) demonstrated the patterns of chromosomal alterations. Patients with a high CIN70 score (white to black scale) had a higher number of copy number alterations (white to blue scale; Spearman's correlation coefficient $(r)=0.28, p<0.001)$ and a higher percentage of genome altered (white to green scale; $r=0.19, p=0.016$ ). Interestingly, the number of mutations (white to red scale) was negatively correlated with the CIN70 score, whereby patients with higher CIN70 scores had fewer mutations $(r=-0.38, p=0.018)$; however, there were a significant number of patients with missing values for this parameter.

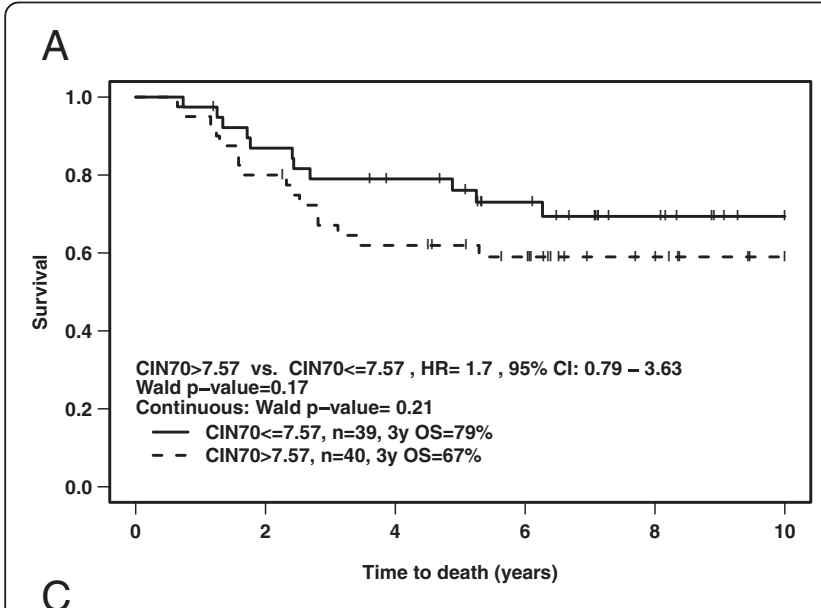

\section{B}
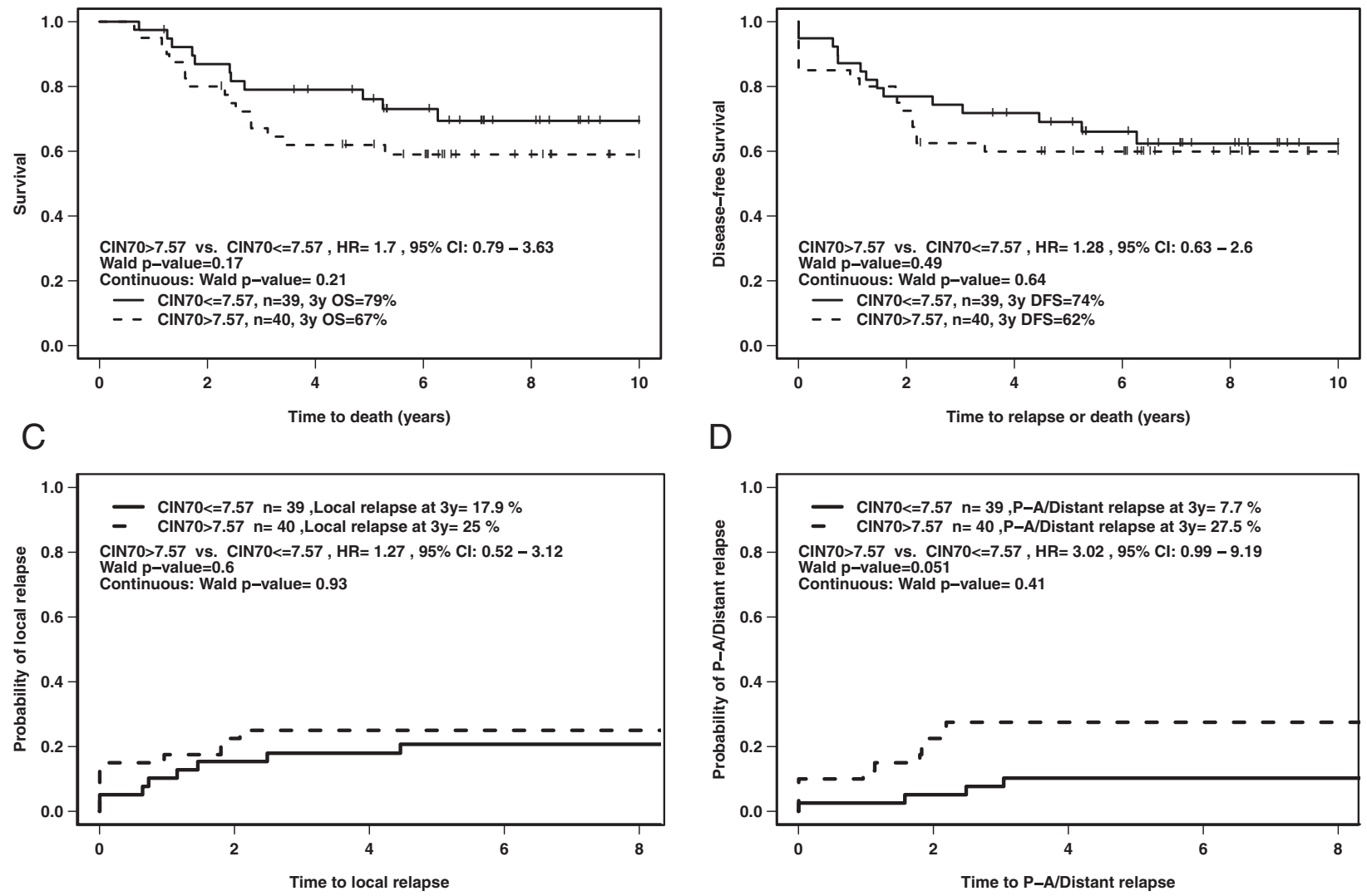

Figure 4 Kaplan-Meier plot of the 79 PM Cancer Centre cervical cancer patients according to CIN70 score. A risk score was calculated for each patient using the CIN70 signature. The median risk score was used to divide patients into high vs. low risk groups. Kaplan-Meier curves are shown for: A) overall survival; B) disease-free survival; C) local relapse; D) para-aortic or distant relapse. HR; hazard ratio, Cl; $95 \%$ confidence interval, P-A; para-aortic. 


\section{CIN70 score was related to distant relapse in cervical cancer patients}

The previously published CIN70 signature [14] was used to calculate the risk score for each patient $(n=79)$ and the median risk score was used to dichotomize a low $v s$. high-risk group. According to the Kaplan-Meier analysis, the CIN70 signature achieved borderline significance for para-aortic nodal or distant relapse (Figure 4D), with a hazard ratio of 3.02 and Wald p-value of 0.05 , but not significant for OS, DFS, or local relapse (Figure 4A-C).

\section{Discussion}

Previous studies have shown that CIN is a hallmark of most solid and many hematopoietic human cancers [2,8,10,11,34-37]. Various mechanisms of CIN have been described, including centrosome duplication [38-40], spindle assembly checkpoint defects [41,42], telomere dysfunction [43], faulty cell-cycle regulation [44-46], sister chromatid cohesion defects $[47,48]$, and the regulation of microtubule attachments to chromosomes at kinetochores [49-51]. To date, CIN is rarely measured in cancer patients, due to technical complexity and lack of a clear understanding of the clinical value of CIN [12].

The CIN70 signature was developed by Carter et al. using a computational method to identify specific genes that were expressed in correlation with total functional aneuploidy, across multiple types of cancer [14]. Overexpression of this signature was shown to predict clinical outcome in six types of cancer, and was also able to stratify grade 1 and 2 breast tumours according to clinical outcome. Birkbak et al. reported that extremes of CIN score were associated with poor prognosis in four types of cancer (breast, ovarian, gastric, and non-small cell lung) [52]. Our study further extended the application of this CIN70 signature to cervical cancer. However, we failed to recapitulate the non-monotonic relationship of CIN with survival as reported in the Birbak et al. study. This could be due to the fact that our study was underpowered to detect a difference in survival, and we examined distant relapse instead of survival. Interestingly, we did observe an inverse relationship between CIN score/copy-number variation and the number of mutations in our cervical cancer samples. This confirms the reported data from Ciriello et al., who analyzed global copy number variation and number of mutations across twelve different cancer types [53]. Our study, along with the others discussed above, are a step towards establishing CIN-associated molecular markers that can be measured in the clinic, and help expand the prognostic utility of CIN in a broad range of human malignancies. Further work should be conducted to determine if the CIN70 signature holds clinical value for other types of cancers, in addition to cervical cancer and the six cancers validated in the Carter et al. study.

\section{Conclusions}

In summary, this study was the first to evaluate the previously published CIN70 signature in cervical cancer patients. CIN70 score was shown to be significantly associated with chromosomal alterations and paraaortic distant relapse. The Carter et al. study was the first step towards establishing CIN-associated molecular markers that could be measured in clinical specimens. Our study further extended the application of this CIN70 signature, and demonstrated that it was associated with para-aortic nodal, as well as distant relapse in patients with cervical cancer. Once longer follow-up is available for the TCGA cohort, it would be important to corroborate the prognostic value of this CIN70 signature for this independent group of patients.

\section{Abbreviations}

CIN: Chromosomal instability; TCGA: The Cancer Genome Atlas; Gy: Gray; FIGO: International Federation of Gynecologists and Obstetricians;

CT: Computed tomography; MRI: Magnetic resonance imaging; OCT: Optimal cutting temperature; H\&E: Hematoxylin and eosin; OS: Overall survival; DFS: Disease-free survival.

\section{Competing interests}

The authors declare that they have no competing interests.

\section{Authors' contributions}

$\mathrm{CH}$ conceived and designed the study, processed the samples, and drafted the manuscript. JB and JS participated in study design, performed the bioinformatic analyses, and drafted the manuscript. MP performed the statistical analyses. BHK and $\mathrm{AH}$ participated in the study design and supervised research. BAC performed pathology analyses. DWH, RPH and MM provided the samples. AF provided samples and supervised research. FFL conceived and designed the study, and supervised the research. All authors read and approved the final manuscript.

\section{Acknowledgements}

This work has been supported by a grant from the Ontario Institute of Cancer Research. CH was supported by a NSERC Postgraduate Scholarship, an Ontario Graduate Scholarship, and the CIHR Terry Fox Foundation Strategic Training Initiative for Excellence in Radiation Research in the $21^{\text {st }}$ Century. Support has also in part been provided from the Campbell Family Institute of Cancer Research, and the Ministry of Health and Long-Term Planning. The authors thank Ms. Trudee Nicklee for preparing the cervix tissue samples.

\section{Author details}

${ }^{1}$ Princess Margaret Cancer Centre, University Health Network, Toronto, ON, Canada. ${ }^{2}$ Department of Radiation Oncology, Princess Margaret Cancer Centre, University Health Network, Toronto, ON, Canada. ${ }^{3}$ Department of Radiation Oncology, University of Toronto, Toronto, ON, Canada. ${ }^{4}$ Division of Biostatistics, Princess Margaret Cancer Centre, University Health Network, Toronto, ON, Canada. ${ }^{5}$ Department of Pathology, University Health Network, Toronto, ON, Canada. ${ }^{6}$ Division of Medical Oncology, Princess Margaret Cancer Centre, Toronto, ON, Canada. ${ }^{7}$ Medical Biophysics Department, University of Toronto, Toronto, ON, Canada.

Received: 29 January 2015 Accepted: 27 April 2015

Published online: 06 May 2015

\section{References}

1. Lengauer C, Kinzler KW, Vogelstein B. Genetic instabilities in human cancers. Nature. 1998;396:643-9.

2. Cimini D. Merotelic kinetochore orientation, aneuploidy, and cancer. Biochim Biophys Acta. 2008;1786:32-40. 
3. Boveri T. Concerning the origin of malignant tumours by Theodor Boveri. Translated and annotated by Henry Harris. J Cell Sci. 2008;121 Suppl 1:1-84.

4. Schvartzman JM, Sotillo R, Benezra R. Mitotic chromosomal instability and cancer: mouse modelling of the human disease. Nat Rev Cancer. 2010;10:102-15.

5. Weaver BA, Silk AD, Montagna C, Verdier-Pinard P, Cleveland DW. Aneuploidy acts both oncogenically and as a tumor suppressor. Cancer Cell. 2007;11:25-36.

6. Diaz-Rodriguez E, Sotillo R, Schvartzman JM, Benezra R. Hec1 overexpression hyperactivates the mitotic checkpoint and induces tumor formation in vivo. Proc Natl Acad Sci U S A. 2008;105:16719-24.

7. Sotillo R, Schvartzman JM, Socci ND, Benezra R. Mad2-induced chromosome instability leads to lung tumour relapse after oncogene withdrawal. Nature. 2010;464:436-40.

8. Walther A, Houlston R, Tomlinson I. Association between chromosomal instability and prognosis in colorectal cancer: a meta-analysis. Gut. 2008:57:941-50.

9. Florl AR, Schulz WA. Chromosomal instability in bladder cancer. Arch Toxicol. 2008:82:173-82.

10. M'Kacher R, Andreoletti L, Flamant S, Milliat F, Girinsky T, Dossou J, et al. JC human polyomavirus is associated to chromosomal instability in peripheral blood lymphocytes of Hodgkin's lymphoma patients and poor clinical outcome. Ann Oncol. 2010;21:826-32.

11. van de Wetering $\mathrm{Cl}$, Horne MC, Knudson CM. Chromosomal instability and supernumerary centrosomes represent precursor defects in a mouse model of T-cell lymphoma. Cancer Res. 2007;67:8081-8.

12. Diaz Jr LA. The current clinical value of genomic instability. Semin Cancer Biol. 2005;15:67-71.

13. Bakhoum SF, Compton DA. Chromosomal instability and cancer: a complex relationship with therapeutic potential. J Clin Invest. 2012;122:1138-43.

14. Carter SL, Eklund AC, Kohane IS, Harris LN, Szallasi Z. A signature of chromosomal instability inferred from gene expression profiles predicts clinical outcome in multiple human cancers. Nat Genet. 2006;38:1043-8.

15. Nutt CL, Mani DR, Betensky RA, Tamayo P, Cairncross JG, Ladd C, et al. Gene expression-based classification of malignant gliomas correlates better with survival than histological classification. Cancer Res. 2003;63:1602-7.

16. Freije WA, Castro-Vargas FE, Fang Z, Horvath S, Cloughesy T, Liau LM, et al. Gene expression profiling of gliomas strongly predicts survival. Cancer Res. 2004;64:6503-10.

17. Phillips HS, Kharbanda S, Chen R, Forrest WF, Soriano RH, Wu TD, et al. Molecular subclasses of high-grade glioma predict prognosis, delineate a pattern of disease progression, and resemble stages in neurogenesis. Cancer Cell. 2006;9:157-73.

18. Wang Y, Klijn JG, Zhang Y, Sieuwerts AM, Look MP, Yang F, et al. Gene-expression profiles to predict distant metastasis of lymph-node-negative primary breast cancer. Lancet. 2005:365:671-9.

19. Sotiriou C, Wirapati P, Loi S, Harris A, Fox S, Smeds J, et al. Gene expression profiling in breast cancer: understanding the molecular basis of histologic grade to improve prognosis. J Natl Cancer Inst. 2006;98:262-72.

20. van de Vijver MJ, He YD, van't Veer LJ, Dai H, Hart AA, Voskuil DW, et al. A gene-expression signature as a predictor of survival in breast cancer. N Engl J Med. 2002;347:1999-2009.

21. Veer $L J \vee$ 't, Dai $H$, van de Vijver MJ, He YD, Hart AA, Mao M, et al. Gene expression profiling predicts clinical outcome of breast cancer. Nature. 2002;415:530-6

22. Bild AH, Yao G, Chang JT, Wang Q, Potti A, Chasse D, et al. Oncogenic pathway signatures in human cancers as a guide to targeted therapies. Nature. 2006;439:353-7.

23. Bhattacharjee A, Richards WG, Staunton J, Li C, Monti S, Vasa P, et al. Classification of human lung carcinomas by mRNA expression profiling reveals distinct adenocarcinoma subclasses. Proc Natl Acad Sci U S A. 2001;98:13790-5.

24. Lopez-Rios F, Chuai S, Flores R, Shimizu S, Ohno T, Wakahara K, et al. Global gene expression profiling of pleural mesotheliomas: overexpression of aurora kinases and P16/CDKN2A deletion as prognostic factors and critical evaluation of microarray-based prognostic prediction. Cancer Res. 2006;66:2970-9.

25. Pomeroy SL, Tamayo P, Gaasenbeek M, Sturla LM, Angelo M, McLaughlin $M E$, et al. Prediction of central nervous system embryonal tumour outcome based on gene expression. Nature. 2002:415:436-42.

26. Shipp MA, Ross KN, Tamayo P, Weng AP, Kutok JL, Aguiar RC, et al. Diffuse large B-cell lymphoma outcome prediction by gene-expression profiling and supervised machine learning. Nat Med. 2002;8:68-74.

27. Jemal A, Bray F, Center MM, Ferlay J, Ward E, Forman D. Global cancer statistics. CA Cancer J Clin. 2011;61:69-90.
28. Howlader N, Noone A, Krapcho M, Garshell J, Miller D, Altekruse S, et al. SEER Cancer Statistics Review, 1975-2011. Bethesda, MD: National Cancer Institute; 2014. http://seer.cancer.gov/csr/1975_2011/. Accessed 6 April 2015.

29. Waggoner SE. Cervical cancer. Lancet. 2003;361:2217-25.

30. Irizarry RA, Hobbs B, Collin F, Beazer-Barclay YD, Antonellis KJ, Scherf U, et al. Exploration, normalization, and summaries of high density oligonucleotide array probe level data. Biostatistics. 2003:4:249-64.

31. Gautier L, Cope L, Bolstad BM, Irizarry RA. affy-analysis of Affymetrix GeneChip data at the probe level. Bioinformatics. 2004;20:307-15.

32. Bandla S, Pennathur A, Luketich JD, Beer DG, Lin L, Bass AJ, et al, Comparative genomics of esophageal adenocarcinoma and squamous cell carcinoma. Ann Thorac Surg. 2012;93:1101-6.

33. Tagawa H, Karnan S, Suzuki R, Matsuo K, Zhang X, Ota A, et al. Genome-wide array-based CGH for mantle cell lymphoma: identification of homozygous deletions of the proapoptotic gene BIM. Oncogene. 2005;24:1348-58.

34. Pino MS, Chung DC. The chromosomal instability pathway in colon cancer. Gastroenterology. 2010;138:2059-72

35. Lingle WL, Lukasiewicz K, Salisbury JL. Deregulation of the centrosome cycle and the origin of chromosomal instability in cancer. Adv Exp Med Biol. 2005;570:393-421.

36. Kramer A, Schweizer S, Neben K, Giesecke C, Kalla J, Katzenberger T, et al. Centrosome aberrations as a possible mechanism for chromosomal instability in non-Hodgkin's lymphoma. Leukemia. 2003;17:2207-13.

37. Swanton C, Tomlinson I, Downward J. Chromosomal instability, colorectal cancer and taxane resistance. Cell Cycle. 2006;5:818-23.

38. Lentini L, Amato A, Schillaci T, Di Leonardo A. Simultaneous Aurora-A/STK15 overexpression and centrosome amplification induce chromosomal instability in tumour cells with a MIN phenotype. BMC Cancer. 2007;7:212.

39. Silkworth WT, Nardi IK, Scholl LM, Cimini D. Multipolar spindle pole coalescence is a major source of kinetochore mis-attachment and chromosome mis-segregation in cancer cells. PLoS One. 2009;4:e6564.

40. Ganem NJ, Godinho SA, Pellman D. A mechanism linking extra centrosomes to chromosomal instability. Nature. 2009:460:278-82.

41. Cahill DP, Lengauer C, Yu J, Riggins GJ, Willson JK, Markowitz SD, et al. Mutations of mitotic checkpoint genes in human cancers. Nature. 1998;392:300-3.

42. Michel LS, Liberal V, Chatterjee A, Kirchwegger R, Pasche B, Gerald W, et al. MAD2 haplo-insufficiency causes premature anaphase and chromosome instability in mammalian cells. Nature. 2001;409:355-9.

43. Tusell L, Pampalona J, Soler D, Frias C, Genesca A. Different outcomes of telomere-dependent anaphase bridges. Biochem Soc Trans. 2010;38:1698-703.

44. Rajagopalan H, Jallepalli PV, Rago C, Velculescu VE, Kinzler KW, Vogelstein B, et al. Inactivation of hCDC4 can cause chromosomal instability. Nature. 2004:428:77-81.

45. Sotillo R, Hernando E, Diaz-Rodriguez E, Teruya-Feldstein J, Cordon-Cardo C, Lowe SW, et al. Mad2 overexpression promotes aneuploidy and tumorigenesis in mice. Cancer Cell. 2007;11:9-23.

46. Hernando E, Nahle Z, Juan G, Diaz-Rodriguez E, Alaminos M, Hemann M, et al. Rb inactivation promotes genomic instability by uncoupling cell cycle progression from mitotic control. Nature. 2004;430:797-802.

47. Zhang N, Ge G, Meyer R, Sethi S, Basu D, Pradhan S, et al. Overexpression of Separase induces aneuploidy and mammary tumorigenesis. Proc Natl Acad Sci U S A. 2008;105:13033-8.

48. Barber TD, McManus K, Yuen KW, Reis M, Parmigiani G, Shen D, et al. Chromatid cohesion defects may underlie chromosome instability in human colorectal cancers. Proc Natl Acad Sci U S A. 2008;105:3443-8.

49. Bakhoum SF, Thompson SL, Manning AL, Compton DA. Genome stability is ensured by temporal control of kinetochore-microtubule dynamics. Nat Cell Biol. 2009;11:27-35.

50. Bakhoum SF, Genovese G, Compton DA. Deviant kinetochore microtubule dynamics underlie chromosomal instability. Curr Biol. 2009;19:1937-42.

51. Cimini D, Wan $X$, Hirel CB, Salmon ED. Aurora kinase promotes turnover of kinetochore microtubules to reduce chromosome segregation errors. Curr Biol. 2006;16:1711-8.

52. Birkbak NJ, Eklund AC, Li Q, McClelland SE, Endesfelder D, Tan P, et al. Paradoxical relationship between chromosomal instability and survival outcome in cancer. Cancer Res. 2011;71:3447-52.

53. Ciriello G, Miller ML, Aksoy BA, Senbabaoglu Y, Schultz N, Sander C. Emerging landscape of oncogenic signatures across human cancers. Nat Genet. 2013;45:1127-33. 